\title{
Extracting gluon condensate from the average plaquette
}

\author{
Taekoon Lee
}

Department of Physics, Kunsan National University, Kunsan 573-701, Korea

\begin{abstract}
The perturbative contribution in the average plaquette is subtracted using Borel summation and the remnant of the plaquette is shown to scale as a dim- 4 condensate. A critical review is presented of the renormalon subtraction scheme that claimed a dim-2 condensate. The extracted gluon condensate is compared with the latest result employing high order (35-loop) calculation in the stochastic perturbation theory.
\end{abstract}

Keywords: renormalon, plaquette, gluon condensate, Borel summation, bilocal expansion

\section{Introduction}

Extracting the gluon condensate from the average plaquette is an old problem of lattice gauge theory. The operator product expansion (OPE) for the plaquette in pure SU(3) Yang-Mills theory is given by

$$
\begin{aligned}
P(\beta) & \equiv\left\langle 1-\frac{1}{3} \operatorname{Tr} \mathrm{U}_{\square}\right\rangle \\
& =\sum_{n=1} \frac{c_{n}}{\beta^{n}}+\frac{\pi^{2}}{36} Z(\beta)\left\langle\frac{\alpha_{s}}{\pi} G G\right\rangle a^{4}+O\left(a^{6}\right),
\end{aligned}
$$

where $\beta$ is the lattice coupling and $a$ the lattice spacing.

To extract the gluon condensate the perturbative contribution, which dominates the plaquette, must be subtracted accurately, to better than one part in $10^{4}$. The perturbative series is expected to be an asymptotic series and at high orders the coefficients to be dominated by renormalon-caused large order behavior. However, the perturbative coefficients computed using stochastic perturbation theory [1] to 10-loop order does not display a renormalon behavior but a power law that grows much faster than the expected renormalon-caused large order behavior.

To subtract the perturbative contribution Burgio et al. [2] introduced a continuum scheme in which the renormalon contribution to the perturbative part of the plaquette is calculated by matching the computed high order coefficients with the renormalon-caused large order behavior mapped to the lattice scheme. This scheme of subtracting perturbative contribution resulted in a power

Email address: tlee@kunsan.ac.kr (Taekoon Lee) correction that scales, surprisingly, as a dim-2 condensate. This is in contradiction with the OPE (1) that demands the leading power correction be of a dim- 4 condensate.

In this presentation based on [3] we give a critical review of the above renormalon subtraction scheme and show that the continuum scheme in which the renormalon was subtracted is far from a renormalondominated scheme and it fails as well the selfconsistency test.

We then introduce a renormalon subtraction scheme based on the bilocal expansion of the Borel transform in continuum scheme, and show that the plaquette data less the Borel-summed perturbative contribution scales as a dim- 4 condensate. The extracted gluon condensate is then compared with the result from the recent high order (35-loop) calculation of the plaquette using stochastic perturbation theory.

\section{Critical review of existing renormalon subtrac- tion scheme}

The renormalon subtraction scheme of Burgio et at. [2] writes essentially the average plaquette as

$$
P(\beta)=P^{\mathrm{ren}}\left(\beta_{c}\right)+\delta P\left(\beta_{c}\right)+P_{\mathrm{NP}}(\beta),
$$

where

$$
P^{\mathrm{ren}}\left(\beta_{c}\right)=\int_{0}^{b_{\max }} e^{-\beta_{c} b} \frac{\mathcal{N}}{\left(1-b / z_{0}\right)^{1+\nu}} d b
$$

with $\beta_{c}$ denoting the coupling in the continuum scheme defined by

$$
\beta_{c}=\beta-r_{1}-\frac{r_{2}}{\beta}
$$


and

$$
z_{0}=\frac{16 \pi^{2}}{33}, \quad v=\frac{204}{121} .
$$

Here the plaquette is divided into the renormalon contribution $P^{\text {ren }}$ and the rest of the perturbative contribution $\delta P$, and nonperturbative correction $P_{\mathrm{NP}}$. The asymptotically divergent behavior of the perturbative series is contained in $P^{\text {ren }}$, and $\delta P$ denotes the rest that can be expressed as a convergent series.

Now define $P_{\mathrm{NP}}^{(N)}$ with

$$
P_{\mathrm{NP}}^{(N)}(\beta) \equiv P(\beta)-P^{\mathrm{ren}}\left(\beta_{c}\right)-\sum_{n=1}^{N}\left(c_{n}-C_{n}^{\mathrm{ren}}\right) \beta^{-n},
$$

where $C_{n}^{\text {ren }}$ denotes the perturbative coefficients in the lattice scheme of $P^{\text {ren }}$. Note that, by definition, $P_{\mathrm{NP}}^{(N)}$ is free of perturbative coefficients to order $N$. The constants $r_{1}, r_{2}$ that define the continuum scheme and the normalization constant $\mathcal{N}$ are to be determined so that $C_{n}^{\text {ren }}$ converges to $c_{n}$ at large orders. In the continuum scheme given by

$$
r_{1}=3.1, \quad r_{2}=2.0,
$$

and with an appropriate value for $\mathcal{N}$ it was observed that $C_{n}^{\text {ren }}$ converge to $c_{n}$ at the orders computed in stochastic perturbation theory. Because the last term in (6) is a convergent series $P_{\mathrm{NP}}^{(N)}$ is well-defined at $N \rightarrow \infty$, and this is precisely the quantity that was assumed to represent the power correction, and it was $P_{\mathrm{NP}}^{(8)}$ that was shown to scale as a dim- 2 condensate.

In this procedure the large order behaviors in the lattice scheme and the continuum scheme are matched using only the high order coefficients. However, this matching of large order behaviors would only work when the perturbative coefficients in both schemes are already in asymptotic regime and follow the pattern of renormalon-caused large order behavior. But, because the computed coefficients in the lattice scheme are far from being in the asymptotic regime and do not follow the renormalon pattern the matching cannot be performed. Therefore, the conclusion of a dimension-2 condensate based on this matching should be reexamined.

One way to check the consistency of the above procedure is to map the computed coefficients in the lattice scheme to the continuum scheme and see if the mapped coefficients follow the pattern of a renormalon behavior. As can be seen in Table 11, however, the coefficients in the continuum scheme are sign-alternating, instead of a factorially growing pattern expected from a renormalon behavior. It shows that the mapping of the perturbative coefficients between the lattice scheme and the continuum scheme of (7) at the orders in consideration are still very sensitive on the low order coefficients, which were ignored in the matching procedure of [2]. It is thus obvious that the continuum scheme of (7) cannot be the right scheme where renormalon can be subtracted reliably.

Table 1: The perturbative coefficients of the average plaquette in the continuum scheme.

\begin{tabular}{cccc} 
scheme. & & & \\
\hline$c_{1}^{\text {cont }}$ & $c_{2}^{\text {cont }}$ & $c_{3}^{\text {cont }}$ & $c_{4}^{\text {cont }}$ \\
2.0 & -4.9792 & 10.613 & -10.200 \\
\hline$c_{5}^{\text {cont }}$ & $c_{6}^{\text {cont }}$ & $c_{7}^{\text {cont }}$ & $c_{8}^{\text {cont }}$ \\
-44.218 & 316.34 & -1096. & 1947. \\
\hline
\end{tabular}

Checking the internal consistency of the subtraction scheme also shows an underlying problem. The nonperturbative term in (2) can be written using (6) as

$$
P_{\mathrm{NP}}(\beta)=P_{\mathrm{NP}}^{(N)}(\beta)-\left[\delta P\left(\beta_{c}\right)-\sum_{n=1}^{N}\left(c_{n}-C_{n}^{\mathrm{ren}}\right) \beta^{-n}\right] .
$$

For $P_{\mathrm{NP}}^{(N)}$ to approximate the power correction

$$
\left|\delta P\left(\beta_{c}\right)-\sum_{n=1}^{N}\left(c_{n}-C_{n}^{\mathrm{ren}}\right) \beta^{-n}\right| \ll P_{\mathrm{NP}}^{(N)}(\beta)
$$

must be satisfied. Because $\delta P\left(\beta_{c}\right)$ is by definition a convergent quantity it can be written in a series expansion

$$
\delta P\left(\beta_{c}\right) \equiv \sum_{n=1}^{\infty} D_{n} \beta_{c}^{-n}
$$

where $D_{n}$ can be computed to the order $c_{n}$ are known, and (9) can be written approximately as

$$
\frac{\left|\sum_{n=1}^{N} D_{n} \beta_{c}^{-n}-\sum_{n=1}^{N}\left(c_{n}-C_{n}^{\mathrm{ren}}\right) \beta^{-n}\right|}{P_{\mathrm{NP}}^{(N)}(\beta)} \ll 1 .
$$

In the continuum scheme of (7), and at $N=8$ and $\beta=6.0,6.2$ and 6.4, for example, the ratios are 69,59 and 42 , respectively, which is a severe violation of the consistency condition. This again confirms that the continuum scheme of (7) cannot be a proper scheme for renormalon subtraction.

\section{Renormalon subtraction by Borel summation}

Clearly, the perturbative contribution in the plaquette cannot be subtracted by matching the renormalonbased coefficients in a continuum scheme to the lattice scheme. Instead, one must map the perturbative coefficients in the lattice scheme to a continuum scheme and 
search for a scheme in which the mapped coefficients display a renormalon pattern. Once such a scheme is found one can then use Borel summation in that scheme to subtract the perturbative contribution to extract the nonperturbative power correction.

In this paper we shall assume that such a scheme exists and perform Borel summation using the bilocal expansion of Borel transform [4]. To Borel-sum the divergent perturbative series accurately, one must have a precise description of the Borel transform in the domain that contains the origin as well as the first renormalon singularity in the Borel plane. The bilocal expansion, utilizing the known perturbative coefficients and the properties of the first renormalon singularity, reconstructs the Borel transform in the above domain of interest by interpolating the expansions about the origin and about the renormalon singularity. The Borel transform in the form of bilocal expansion implements the correct nature of the first renormalon singularity as well as the perturbative coefficients that match the known coefficients. The sum of the Borel-summed perturbative contribution and a dim- 4 power correction is then fitted to the plaquette data. A good fit would then suggest the power correction be of dim-4 type.

The Borel summation using bilocal expansion in the continuum scheme using the first $N$-loop perturbations of the plaquette is given in the form:

$$
P_{\mathrm{BR}}^{\mathrm{N}}(\beta)=\operatorname{Re} \int_{0}^{\infty} e^{-\beta_{c} b}\left[\sum_{n=0}^{N-1} \frac{h_{n}}{n !} b^{n}+\frac{\mathcal{N}}{\left(1-b / z_{0}\right)^{1+v}}\right] d b,
$$

where the integration is performed along the real axis on the upper-half plane. The essential idea of the bilocal expansion is to interpolate the two perturbative expansions about the origin and about the renormalon singularity to rebuild the Borel transform. Incorporating the renormalon singularity explicitly extends the applicable domain of the Borel transform from near the origin to areas beyond the renormalon singularity. This scheme was used in summing the perturbative series for the static inter-quark potential as well as the heavy quark pole mass [4, 5]; The Borel-summed static potential agrees very well with lattice results and the convergence in the pole mass case is extremely fast.

$\mathcal{N}$ in (12) denotes the normalization constant of the large order behavior and the coefficients $h_{n}$ are determined so that the Borel transform reproduce the perturbative coefficients in the continuum scheme when expanded about the origin. Thus $h_{n}$ depends on the continuum perturbative coefficients as well as $\mathcal{N}$. By definition, $P_{\mathrm{BR}}^{(N)}(\beta)$, when expanded in $1 / \beta$, reproduces the perturbative coefficients of the average plaquette to the
$N$-loop order that were employed in building the Borel transform. The power correction can then be defined by:

$$
P_{\mathrm{NP}}^{(\mathrm{N})}(\beta) \equiv P(\beta)-P_{\mathrm{BR}}^{(\mathrm{N})}(\beta),
$$

which, by definition, is free of perturbative coefficients to $\operatorname{order} N$.

Using the perturbation to 10-loop order of the plaquette we compute $P_{\mathrm{BR}}^{(10)}(\beta)$ in the continuum scheme defined by (4). The normalization $\mathcal{N}$ is treated as a fitting parameter, and in our scheme the parameters to be fitted are $r_{1}, r_{2}$, and $\mathcal{N}$.

Using the plaquette data for $6.0 \leq \beta \leq 6.8$ from [6] and the relation between the lattice spacing $a$ and $\beta$ from static quark force simulation [7]

$$
\begin{aligned}
\log \left(a / r_{0}\right)= & -1.6804-1.7331(\beta-6)+ \\
& 0.7849(\beta-6)^{2}-0.4428(\beta-6)^{3}
\end{aligned}
$$

the fit gives $\mathcal{N}=165$ and

$$
r_{1}=1.611, \quad r_{2}=0.246,
$$

which values are substantially different from those in (7). The result of the fit is shown in Fig. 11 which shows that the power correction is consistent with a dim- 4 condensate. The agreement improves as $\beta$ increases, albeit with larger uncertainties; The deviation at low $\beta(\beta<6)$ may be attributed to a dim- 6 condensate, which may be seen, though not presented here, by that adding a dim6 power correction in the fit improves the agreement in the whole range of the plot. The error bars are from the uncertainty in the simulated perturbative coefficients of the plaquette. The uncertainty in the normalization constant does not appear to be large: for example, a variation of $20 \%$ in $\mathcal{N}$ causes less than a quarter of those by the perturbative coefficients.

From the fit we obtain a dim-4 power correction of $P_{\mathrm{NP}} \approx 1.6\left(a / r_{0}\right)^{4}$. Because of the asymptotic nature of the perturbative series the power correction of the plaquette is dependent on the subtraction scheme of the perturbative contribution, and thus our result may not be directly compared to those from other subtraction schemes. Nevertheless, it is still interesting to observe that the result is roughly consistent with $0.4\left(a / r_{0}\right)^{4}$ of [8] and $0.7\left(a / r_{0}\right)^{4}$ of [9]. Our result turns out to be a little larger than those estimates; This may be partly accounted for by the fact that the existing results were from the fit in the low $\beta$ range of $\beta \lesssim 6$, in which range the data are below our fitted curve.

\section{Comparison with latest result from 35-loop calcu- lation}

Recently Bali et al. [10, 11] computed the plaquette to 35-loop order using stochastic perturbation theory and 


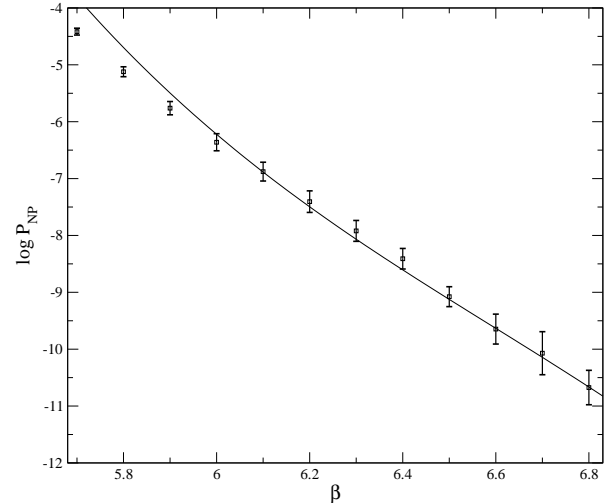

Figure 1: $\log P_{\mathrm{NP}}$ vs. $\beta$. The solid line is for $4 \log \left(a / r_{0}\right)+0.5$. The plot shows the power correction should be of dim- 4 type.

observed a renormalon behavior of the coefficients at high orders, determining the normalization of the large order behavior. With the perturbative expansion in the asymptotic regime the power correction may as well be extracted by subtracting from the average plaquette the perturbative series truncated at the order the loop correction becomes minimal. The power correction thus obtained was shown to be of dim-4 in accordance with the OPE (1), and the gluon condensate was determined to be

$$
\left\langle\frac{\alpha_{s}}{\pi} G G\right\rangle \approx 0.077 \mathrm{GeV}^{4}
$$

with an intrinsic uncertainty of $0.087 \mathrm{GeV}^{4}$. The normalization of the large order behavior in the lattice scheme was obtained as

$$
\mathcal{N}_{\text {lat }}=4.2( \pm 1.7) \times 10^{5} \text {. }
$$

It is interesting to compare these numbers with our results. From the fit in the previous section we obtain the gluon condensate:

$$
\left\langle\frac{\alpha_{s}}{\pi} G G\right\rangle=\frac{36}{\pi^{2}} e^{0.5} r_{0}^{-4} \approx 0.14 \mathrm{GeV}^{4},
$$

and the normalization $\mathcal{N}=165 \pm 50$ (30\% uncertainty) in the continuum scheme corresponds to

$$
\mathcal{N}_{\text {lat }}=\frac{2 \pi}{3} e^{z_{0} r_{1}} \mathcal{N}=7.6( \pm 2.3) \times 10^{5},
$$

which is, remarkably, consistent with (17). Comparing the gluon condensates (16) and (18) we see that they are in agreement within the intrinsic uncertainty. The gluon condensate is dependent on the prescription for the perturbative contribution in the plaquette, and the difference between the two condensate values may be because they came from different prescriptions: Borel summation and truncated power series, respectively.

\section{Conclusions}

The renormalon subtraction procedure of [2] that led to a dim-2 condensate in the plaquette was reexamined. It is found that the continuum scheme employed in the procedure is far from a renormalon-dominated scheme and the procedure also fails a consistency check. As a consequence the power correction extracted is severely contaminated by perturbative contribution, to discredit the claimed dim-2 condensate.

We then introduced a renormalon subtraction scheme that avoids the problems, in which the perturbative contribution is obtained by Borel-summing the perturbative series in a continuum scheme, employing the rebuilt Borel transform in the framework of the bilocal expansion. The power correction obtained in this procedure is of dim-4, in accordance with the OPE of the plaquette. The normalization of the large order behavior of the plaquette as well as the gluon condensate extracted are shown to be in agreement with the latest results from 35-loop order calculations.

\section{Acknowledgements}

I am thankful to S. Han and G. Bali for useful conversations. This research was supported by Basic Science Research Program through the National Research Foundation of Korea (NRF), funded by the Ministry of Education, Science, and Technology (2012R1A1A2044543).

\section{References}

[1] Di Renzo and Scorzato, JHEP 10 (2001) 038

[2] Burgio, Di Renzo, Marchesini, and Onofri, Phys. Lett.B422 (1998) 219.

[3] T. Lee, Phys.Rev. D82 (2010) 114021

[4] T. Lee, Phys.Rev. D67 (2003) 014020.

[5] T. Lee, JHEP 10 (2003) 044.

[6] Boyd et al., Nucl. Phys. 469 (1996) 419.

[7] Necco and Sommer, Nucl. Phys. 622 (2002) 328

[8] P.E.L. Rakow, Pos LAT2005 (2006) 284

[9] Y. Meurice, Phys.Rev. D74 (2003) 096005

[10] G. Bali, C. Bauer, and A. Pineda, Phys.Rev.D89 (2014) 054505 .

[11] G. Bali, C. Bauer, and A. Pineda, arXiv:1403.6477. 